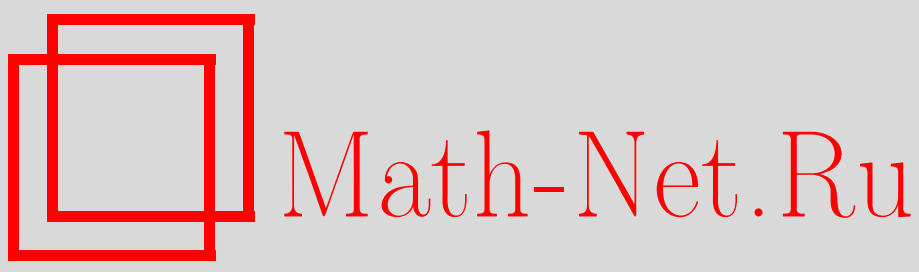

N. V. Pertsev, Exponential decay estimates for some components of solutions to the nonlinear delay differential equations of the living system models, Sibirsk. Mat. Zh., 2020, Volume 61, Number 4, 901-912

DOI: https://doi.org/10.33048/smzh.2020.61.412

Use of the all-Russian mathematical portal Math-Net.Ru implies that you have read and agreed to these terms of use http://www . mathnet.ru/eng/agreement

Download details:

IP : 54.205 .225 .156

April 26, 2023, 16:31:01 
Сибирский математический журнал Июль-август, 2020. Том 61, № 4

\title{
ЭКСПОНЕНЦИАЛЬНО УБЫВАЮЩИЕ ОЦЕНКИ ПО ЧАСТИ КОМПОНЕНТ РЕШЕНИЙ НЕЛИНЕЙНЫХ ДИФФЕРЕНЦИАЛЬНЫХ УРАВНЕНИЙ С ЗАПАЗДЫВАНИЕМ, ВОЗНИКАЮЩИХ В МОДЕЛЯХ ЖИВЫХ СИСТЕМ
}

\author{
Н. В. Перцев
}

\begin{abstract}
Аннотация. Исследуется поведение решений задачи Коши для семейства нелинейных функционально-дифференциальных уравнений с запаздыванием, возникающего в моделях живых систем. Установлена совокупность условий, обеспечивающих экспоненциально убывающие оценки по части компонент решений изучаемой задачи Коши. Параметры экспоненциальных оценок находятся как решение нелинейной системы неравенств, построенной на основе мажорант отображений, входящих в правые части рассматриваемой системы дифференциальных уравнений. Представлены результаты построения экспоненциальных оценок переменных модели, описывающей динамику эпидемического процесса.
\end{abstract}

DOI $10.33048 /$ smzh.2020.61.412

Ключевые слова: функционально-дифференциальное уравнение с запаздыванием, задача Коши, глобальная разрешимость, неотрицательность решений, экспоненциальные убывающие оценки решений, М-матрица, математическая биология, живые системы, эпидемиология.

Введение. Многие математические модели, описывающие динамику живых систем, могут быть представлены в виде задачи Коши

$$
\begin{gathered}
\frac{d x(t)}{d t}=f\left(t, x_{t}\right)-\left(\mu+g\left(t, x_{t}\right)\right) x(t), \quad t \geq 0, \\
x(t)=\psi(t), \quad t \in I_{\omega}=[-\omega, 0]
\end{gathered}
$$

для системы функционально-дифференциальных уравнений с запаздыванием. В уравнениях системы (1) и начальных условиях (2) использованы обозначения

$$
\begin{gathered}
x(t)=\left(x_{1}(t), \ldots, x_{m}(t)\right)^{T}, \quad \psi(t)=\left(\psi_{1}(t), \ldots, \psi_{m}(t)\right)^{T}, \\
f\left(t, x_{t}\right)=\left(f_{1}\left(t, x_{t}\right), \ldots, f_{m}\left(t, x_{t}\right)\right)^{T}, \quad g\left(t, x_{t}\right)=\operatorname{diag}\left(g_{1}\left(t, x_{t}\right), \ldots, g_{m}\left(t, x_{t}\right)\right), \\
\mu=\operatorname{diag}\left(\mu_{1}, \ldots, \mu_{m}\right), \quad m \geq 2,
\end{gathered}
$$

где $x(t)$ - искомая функция, запаздывающая переменная $x_{t}: I_{\omega} \rightarrow R^{m}$ определена по правилу: $x_{t}(\theta)=x(t+\theta), \theta \in I_{\omega}$, для каждого фиксированного $t \geq 0$, $\psi(t)$ - начальная функция, $f_{i}\left(t, x_{t}\right), g_{i}\left(t, x_{t}\right)$ - некоторые отображения, $\mu_{i}-$

Работа выполнена при финансовой поддержке Российского фонда фундаментальных исследований, проект № 18-29-10086.

(c) 2020 Перцев Н. В. 
константы, $1 \leq i \leq m$, под $d x(t) / d t$ понимается правосторонняя производная (покомпонентно).

В приложениях функция $x(t)$ может означать численность элементов некоторой живой системы (клетки, вирусы, бактерии, микроорганизмы, индивидуумы различных типов или групп и т. д.). Для фиксированного $1 \leq i \leq m$ отображение $f_{i}\left(t, x_{t}\right)$ описывает скорость появления новых элементов $i$-го типа, отображение $g_{i}\left(t, x_{t}\right)$ таково, что $g_{i}\left(t, x_{t}\right) x_{i}(t)$ задает скорость гибели или превращения элементов $i$-го типа за счет взаимодействия элементов между собой, а также под влиянием внешней среды. Выражение $\mu_{i} x_{i}(t)$ означает скорость естественной смертности или миграционного оттока элементов $i$-го типа, а также скорость перехода элементов $i$-го типа между группами.

В [1-3] исследованы решения $x(t)$ задачи Коши $(1),(2)$ для линейных и нелинейных систем уравнений (1). В указанных работах при выполнении ряда условий показана неотрицательность $x(t)$ и предложен подход к построению покомпонентных верхних оценок для $x(t)$. Важной особенностью некоторых нелинейных моделей является структура уравнений системы (1), которые можно записать в виде двух блоков. В таких системах правые части уравнений первого и второго блоков содержат компоненты отображения $f\left(t, x_{t}\right)$, допускающие верхние оценки в виде линейных отображений или констант. Наличие таких оценок позволяет использовать хорошо развитую теорию монотонных операторов [4, гл. 1 , п. $3 ; 5 ; 6$, гл. 2 , п. 2.3 ; гл. 7 , п. 7.1] и построить оценки для компонент решения $x(t)$ задачи Коши $(1),(2)$.

Целью настоящей работы является получение условий, обеспечивающих существование экспоненциально убывающих оценок по части компонент решения $x(t)$ задачи Коши $(1),(2)$ на промежутке $[0, \infty)$.

1. Основные предположения. Используем обозначения и предположения, введенные в [3]. Пусть $\|v\|_{R^{m}}=\sum_{i=1}^{m}\left|v_{i}\right|$ - норма вектора $v \in R^{m}$. Положим $J=[a, b] \subset R, A \subseteq R^{m}$. Через $C(J, A)$ обозначим множество всех непрерывных функций $z: J \rightarrow A$ с нормой

$$
\|z\|=\max _{\theta \in J}\left(\|z(\theta)\|_{R^{m}}\right), \quad z \in C\left(J, R^{m}\right) .
$$

Обозначим через $B_{d}=\left\{z \in C\left(I_{\omega}, R^{m}\right):\|z\| \leq d\right\}$ шар в пространстве $C\left(I_{\omega}, R^{m}\right)$. Для $u, w \in R^{m}$ неравенства $u<0, u>0, u \leq w, u \geq w$ понимаются покомпонентно. Если $x, y \in C(J, A)$, то для каждого $t \in \bar{J}$ неравенство $x(t) \leq y(t)$ понимается как неравенство между соответствующими векторами.

Примем, что отображения, функции и константы, входящие в (1), (2), удовлетворяют для каждого $1 \leq i \leq m$ набору приведенных ниже предположений, которые обозначим через (Н0):

1) $f_{i}, g_{i}: R_{+} \times C\left(I_{\omega}, A_{\xi}\right) \rightarrow R$, где $A_{\xi}=\left\{u \in R^{m}: u \geq \xi\right\}, \xi \in R^{m}, \xi<0-$ некоторый фиксированный вектор;

2) $f_{i}, g_{i}: R_{+} \times C\left(I_{\omega}, R_{+}^{m}\right) \rightarrow R_{+}$;

3) $f_{i}(t, z), g_{i}(t, z)$ непрерывны по $(t, z) \in R_{+} \times C\left(I_{\omega}, A_{\xi}\right)$ и локально липшицевы по $z$ : для каждого $d \in R, d>0$, существуют константы $L_{f}^{(i)}=L_{f}^{(i)}(\xi, d)>0$, $L_{g}^{(i)}=L_{g}^{(i)}(\xi, d)>0$ такие, что при всех $z_{1}, z_{2} \in B_{d} \cap C\left(I_{\omega}, A_{\xi}\right)$ и $t \in[0, \infty)$ выполнены неравенства

$$
\left|f_{i}\left(t, z_{1}\right)-f_{i}\left(t, z_{2}\right)\right| \leq L_{f}^{(i)}\left\|z_{1}-z_{2}\right\|, \quad\left|g_{i}\left(t, z_{1}\right)-g_{i}\left(t, z_{2}\right)\right| \leq L_{g}^{(i)}\left\|z_{1}-z_{2}\right\|
$$


4) $\psi_{i}: I_{\omega} \rightarrow R_{+}-$непрерывная функция;

5) $\mu_{i}>0$.

Следуя [7, гл. 2, п. 2.1; 8, гл. 1, п. 2.1, 2.2], решением задачи Коши (1), (2) на промежутке $[0, \infty)$ будем называть функцию $x(t)$, непрерывную на любом конечном промежутке $I_{\omega} \cup[0, \tau], \tau>0$, имеющую непрерывную производную на промежутке $[0, \tau)$ и удовлетворяющую начальному условию (2) и уравнениям системы (1) для всех $t \in[0, \tau)$. Интегрируя (1) по методу вариации произвольной постоянной с учетом (2), приходим к системе интегральных уравнений, дополненной начальными данными:

$$
\begin{gathered}
x(t)=e^{-\int_{0}^{t}\left(\mu+g\left(s, x_{s}\right)\right) d s} \psi(0)+\int_{0}^{t} e^{-\int_{a}^{t}\left(\mu+g\left(s, x_{s}\right)\right) d s} f\left(a, x_{a}\right) d a, \quad t \geq 0, \\
x(t)=\psi(t), \quad t \in I_{\omega} .
\end{gathered}
$$

В системе уравнений (3) под

$$
e^{-\int_{a}^{t}\left(\mu+g\left(s, x_{s}\right)\right) d s}, \quad 0 \leq a \leq t,
$$

понимается диагональная матрица, построенная на основе диагональной матрицы $\mu+g\left(s, x_{s}\right)$. Решением задачи $(3),(4)$ на промежутке $[0, \infty)$ будем называть функцию $x(t)$, непрерывную на любом конечном промежутке $I_{\omega} \cup[0, \tau], \tau>0$, и удовлетворяющую начальному условию (4) и уравнениям системы (3) для всех $t \in[0, \tau]$. Опираясь на стандартный подход [7, гл. 2, п. 2.1], получаем, что задачи (1), (2) и (3), (4) эквивалентны. Следовательно, для изучения поведения решения $x(t)$ задачи Коши (1), (2) можно воспользоваться системой (3), (4).

В [3] показано, что предположение (Н0), рассматриваемое совместно с дополнительными предположениями относительно отображения $f\left(t, x_{t}\right)$ и элементов диагональной матрицы $g\left(t, x_{t}\right)$, обеспечивает существование, единственность и неотрицательность решения $x(t)$ задачи $(3),(4)$ на промежутке $[0, \infty)$. Кроме того, для компонент решения $x(t)$ построены верхние оценки, вид которых зависит от используемых дополнительных предположений.

Следующий раздел посвящен построению верхних оценок решения $x(t)$ задачи $(3),(4)$, содержащих экспоненциально убывающие оценки для части компонент $x(t)$.

2. Дополнительное предположение и основной результат. Введем обозначения: $k-$ фиксированный индекс, $1 \leq k<m, \eta=\left(\eta_{k+1}, \ldots, \eta_{m}\right)^{T}-$ вектор с неотрицательными компонентами, $D_{\eta}-$ подмножество $R_{+}^{m}$, заданное с помощью вектора $\eta$ :

$$
D_{\eta}=\left\{u \in R_{+}^{m}: 0 \leq u_{j} \leq \eta_{j}, k+1 \leq j \leq m\right\} .
$$

Введем предположение (H1) относительно $f\left(t, x_{t}\right)$. Полагаем, что при некотором $1 \leq k<m$ отображение $f\left(t, x_{t}\right)$ представлено в виде

$$
f\left(t, x_{t}\right)=\left(f_{1}\left(t, x_{t}\right), \ldots, f_{k}\left(t, x_{t}\right), f_{k+1}\left(t, x_{t}\right), \ldots, f_{m}\left(t, x_{t}\right)\right)^{T}
$$

и удовлетворяет следующим условиям:

1) для всех $\left(t, x_{t}\right) \in R_{+} \times C\left(I_{\omega}, R_{+}^{m}\right)$ выполнено неравенство

$$
\left(f_{k+1}\left(t, x_{t}\right), \ldots, f_{m}\left(t, x_{t}\right)\right)^{T} \leq p=\left(p_{k+1}, \ldots, p_{m}\right)^{T},
$$


где $p_{k+1}>0, \ldots, p_{m}>0$ - некоторые константы;

2) вектор $\eta=\left(\eta_{k+1}, \ldots, \eta_{m}\right)^{T}>0$ с компонентами

$$
\eta_{j}=\frac{p_{j}}{\mu_{j}}, \quad k+1 \leq j \leq m,
$$

таков, что для всех $\left(t, x_{t}\right) \in R_{+} \times C\left(I_{\omega}, D_{\eta}\right)$ имеет место оценка

$$
\begin{aligned}
\left(f_{1}\left(t, x_{t}\right), \ldots, f_{k}\left(t, x_{t}\right)\right)^{T} & \leq L\left(x_{t}^{(k)}\right) \\
& =\sum_{i=0}^{n} L_{k, i} x^{(k)}\left(t-\omega_{i}\right)+\int_{-\omega}^{0} L_{k, n+1}(\theta) x^{(k)}(t+\theta) d \theta,
\end{aligned}
$$

где $x^{(k)}(t)=\left(x_{1}(t), \ldots, x_{k}(t)\right)^{T}$, запаздывания $0<\omega_{i} \leq \omega<\infty, 1 \leq i \leq n$, $\omega_{0}=0, L_{k, 0}, \ldots, L_{k, n}, L_{k, n+1}(\theta)$ - неотрицательные $(k \times k)$-матрицы, ненулевые элементы $L_{k, n+1}(\theta)$ интегрируемы по Риману, каждая строка матрицы

$$
L_{[k]}=\sum_{i=0}^{n} L_{k, i}+\int_{-\omega}^{0} L_{k, n+1}(\theta) d \theta
$$

отлична от нулевой.

Опираясь на предположение (H1), положим

$$
\mu_{[k]}=\operatorname{diag}\left(\mu_{1}, \ldots, \mu_{k}\right), \quad I_{[k]}=\operatorname{diag}(1, \ldots, 1), \quad \psi_{[k]}(t)=\left(\psi_{1}(t), \ldots, \psi_{k}(t)\right)^{T} .
$$

Используя выражение для $L\left(x_{t}^{(k)}\right)$ из $(7)$, рассмотрим задачу Коши относительно функции $y(t)=\left(y_{1}(t), \ldots, y_{k}(t)\right)^{T}$ :

$$
\begin{gathered}
\frac{d y(t)}{d t}=L\left(y_{t}\right)-\mu_{[k]} y(t) \\
=\sum_{i=0}^{n} L_{k, i} y\left(t-\omega_{i}\right)+\int_{-\omega}^{0} L_{k, n+1}(\theta) y(t+\theta) d \theta-\mu_{[k]} y(t), \quad t \geq 0, \\
y(t)=\psi_{[k]}(t), t \in I_{\omega} .
\end{gathered}
$$

Решением задачи Коши (9), (10) на промежутке $[0, \infty)$ будем называть функцию $y(t)$, непрерывную на любом конечном промежутке $I_{\omega} \cup[0, \tau], \tau>0$, имеющую непрерывную производную на промежутке $[0, \tau)$ и удовлетворяющую начальному условию (10) и уравнениям системы (9) для всех $t \in[0, \tau)$; при $t=0$ под $d y(t) / d t$ понимается правосторонняя производная (покомпонентно). Интегрируя (9) по методу вариации произвольной постоянной с учетом (10), перейдем к системе линейных интегральных уравнений с заданным начальным условием:

$$
\begin{gathered}
y(t)=e^{-\mu_{[k]} t} \psi(0)+\int_{0}^{t} e^{-\mu_{[k]}(t-a)} L\left(y_{a}\right) d a, \quad t \geq 0, \\
y(t)=\psi_{[k]}(t), \quad t \in I_{\omega} .
\end{gathered}
$$

Решением задачи (11), (12) на промежутке $[0, \infty)$ будем называть функцию $y(t)$, непрерывную на любом конечном промежутке $I_{\omega} \cup[0, \tau], \tau>0$ и удовлетворяющую начальному условию (12) и уравнениям системы (11) для всех $t \in[0, \tau]$. 
Нахождение решения задачи Коши (9), (10) эквивалентно отысканию решения задачи (11), (12).

Заметим, что в рамках предположения (Н1) матрица $L_{[k]}$, заданная формулой (8), неотрицательна, а внедиагональные элементы матрицы $\mu_{[k]}-L_{[k]}$ неположительны. Это означает, что $\mu_{[k]}-L_{[k]}$ относится к матрицам специального вида. Будем говорить, что $\mu_{[k]}-L_{[k]}$ является невырожденной М-матрицей, если она имеет обратную матрицу и обратная матрица $\left(\mu_{[k]}-L_{[k]}\right)^{-1}$ неотрицательна. В [9, гл. $6 ; 10$, гл. 2 , п. 36] приведен ряд критериев, позволяющих проверять матрицу $\mu_{[k]}-L_{[k]}$ на принадлежность семейству невырожденных М-матриц.

Лемма 1. Пусть матрица $L_{[k]}$, введенная в предположении (H1), такова, что $\mu_{[k]}-L_{[k]}$ является невырожденной М-матрицей. Тогда для решения $y(t)$ задачи Коши (9), (10) имеет место оценка

$$
0 \leq y(t) \leq c e^{-r t}, \quad t \in I_{\omega} \cup[0, \infty),
$$

где $c \in R^{k}, r \in R$ удовлетворяют системе неравенств

$$
\begin{array}{cl}
c>0, & \left(\mu_{[k]}-r I_{[k]}-\sum_{i=0}^{n} e^{r \omega_{i}} L_{k, i}-\int_{-\omega}^{0} e^{-r \theta} L_{k, n+1}(\theta) d \theta\right) c \geq 0, \\
c \geq \max _{t \in I_{\omega}}\left(e^{r t} \psi_{[k]}(t)\right), \quad 0<r<\min \left(\mu_{1}, \ldots, \mu_{k}\right) .
\end{array}
$$

ДокАЗАтЕльство. Пусть $c \in R^{k}, r \in R$ - некоторые вектор и константа. Введем функцию

$$
v(t)=c e^{-r t}, \quad t \in I_{\omega} \cup[0, \infty) .
$$

Опираясь на условия леммы, используем теорему 3 из [1] применительно к задаче Коши (9), (10) (задаче (11), (12)). Получаем, что существуют $c \in R^{k}$ и $r \in R$, удовлетворяющие (14), (15) и такие, что для функции $v(t)$, приведенной в (16), верны неравенства

$$
\begin{gathered}
e^{-\mu_{[k]} t} \psi_{[k]}(0)+\int_{0}^{t} e^{-\mu_{[k]}(t-a)} L\left(v_{a}\right) d a \leq v(t), \quad 0 \leq t<\infty, \\
\psi_{[k]}(t) \leq v(t), \quad t \in I_{\omega} .
\end{gathered}
$$

Кроме того, из $(11),(12),(17),(18)$ следует, что при всех $t \in I_{\omega} \cup[0, \infty)$ для решения $y(t)$ задачи Коши (9), (10) верно $0 \leq y(t) \leq v(t)=c \exp (-r t)$, т. е. имеет место оценка (13). Лемма доказана.

Отметим, что способы нахождения $c \in R^{k}$ и $r \in R$, используемых в оценке (13), приведены в разд. 3 из [1] и в разд. 2 из [2].

Перейдем к построению экспоненциально убывающих оценок решения $x(t)$ задачи Коши (1), (2) по первым $k$ компонентам. Воспользуемся подходом, изложенным в [3]. Зафиксируем $\tau>0$. Под $C_{\psi} \subset C\left([-\omega, \tau], R^{m}\right)$ будем понимать множество, состоящее из всех функций $x \in C\left([-\omega, \tau], R^{m}\right)$ таких, что $x(t)=\psi(t), t \in I_{\omega}$. Решением задачи (3), (4) на промежутке $[0, \tau]$ будем называть функцию $x \in C_{\psi}$ такую, что $x(t)$ удовлетворяет (3) для всех $t \in[0, \tau]$.

Примем, что $C_{\psi, 0} \subset C_{\psi}$ - множество, состоящие из функций $x \in C_{\psi}$ таких, что $x(t) \geq 0, t \in[-\omega, \tau]$. Пусть $v=v(t)=\left(v_{1}(t), \ldots, v_{m}(t)\right)^{T}$ - некоторая функция с неотрицательными компонентами, непрерывная на промежутке $[-\omega, \tau]$. 
Положим, что $C_{\psi, 0, v}$ состоит из функций $x \in C_{\psi}$, удовлетворяющих неравенствам $0 \leq x(t) \leq v(t), t \in[-\omega, \tau]$.

Опираясь на соотношения $(3),(4)$, определим оператор $F$, который каждой функции $x \in C_{\psi, 0}$ сопоставляет функцию $F(x) \in C_{\psi, 0}$ по формулам

$$
\begin{gathered}
F(x)(t)=\psi(t), \quad t \in I_{\omega} \\
F(x)(t)=e^{-\int_{0}^{t}\left(\mu+g\left(s, x_{s}\right)\right) d s} \psi(0)+\int_{0}^{t} e^{-\int_{a}^{t}\left(\mu+g\left(s, x_{s}\right)\right) d s} f\left(a, x_{a}\right) d a, \quad t \in[0, \tau] .
\end{gathered}
$$

Лемма 2. Пусть выполнены предположения (H0), (H1), $\mu_{[k]}-L_{[k]}$ является невырожденной М-матрицей и компоненты начальной функции $\psi$ таковы, что

$$
\max _{t \in I_{\omega}} \psi_{j}(t) \leq \eta_{j}, \quad k+1 \leq j \leq m
$$

Тогда существуют $\beta \in R^{m}, r \in R, \beta>0, r>0$, такие, что при

$$
v(t)=\left(\beta_{1} e^{-r t}, \ldots, \beta_{k} e^{-r t}, \beta_{k+1}, \ldots, \beta_{m}\right)^{T}, \quad t \in R
$$

для каждого $\tau>0$ множество $C_{\psi, 0, v}$ является инвариантным для оператора $F$.

ДокАЗАТЕЛЬСТво. Используя условия леммы, положим, что компоненты вектора $v(t)=\left(v_{1}(t), \ldots, v_{k}(t), v_{k+1}(t), \ldots, v_{m}(t)\right)^{T}$, входящего в определение множества $C_{\psi, 0, v}$, имеют вид

$$
\begin{gathered}
v^{(k)}(t)=\left(v_{1}(t), \ldots, v_{k}(t)\right)=\left(c_{1} e^{-r t}, \ldots, c_{k} e^{-r t}\right), \\
\left(v_{k+1}(t), \ldots, v_{m}(t)\right)=\left(\eta_{k+1}, \ldots, \eta_{m}\right), \quad t \in R,
\end{gathered}
$$

где $c=\left(c_{1}, \ldots, c_{k}\right)^{T}, r$ - параметры функции (16), указанной в лемме 1 , константы $\eta_{k+1}, \ldots, \eta_{m}$ заданы формулой (6). Обозначим

$$
\mu_{*}=\operatorname{diag}\left(\mu_{k+1}, \ldots, \mu_{m}\right), \quad \psi_{*}(t)=\left(\psi_{k+1}(t), \ldots, \psi_{m}(t)\right)^{T}, \quad t \in I_{\omega} .
$$

Зафиксируем $\tau>0$. Пусть $x \in C_{\psi, 0, v}$. По условию для всех $t \in I_{\omega}$ верно $\psi_{*}(t) \leq \eta$. Привлекая $(15)$, получаем, что $0 \leq F(x)(t)=\psi(t) \leq v(t), t \in I_{\omega}$.

Представим оператор $F(x)(t)$ в компонентной записи:

$$
F(x)(t)=\left(F_{1}(x)(t), \ldots, F_{k}(x)(t), F_{k+1}(x)(t), \ldots, F_{m}(x)(t)\right)^{T}, \quad t \in I_{\omega} \cup[0, \tau] .
$$

Покажем, что для всех $t \in[0, \tau]$ верно $0 \leq F(x)(t) \leq v(t)$. При построении приведенных ниже оценок учтем неравенства $0 \leq x(t) \leq v(t), t \in[-\omega, \tau]$.

Опираясь на (5) и (6), находим, что

$$
\begin{aligned}
\left(F_{k+1}(x)(t), \ldots, F_{m}(x)(t)\right)^{T} & \\
& \leq e^{-\mu_{*} t} \psi_{*}(0)+\int_{0}^{t} e^{-\mu_{*}(t-a)}\left(f_{k+1}\left(t, x_{a}\right), \ldots, f_{m}\left(t, x_{a}\right)\right)^{T} d a \\
& \leq e^{-\mu_{*} t} \eta+\int_{0}^{t} e^{-\mu_{*}(t-a)} p d a=\eta=\left(v_{k+1}(t), \ldots, v_{m}(t)\right)^{T}, \quad t \in[0, \tau] .
\end{aligned}
$$


Используя (6), (7) и лемму 1 , получаем, что

$$
\begin{aligned}
& \left(F_{1}(x)(t), \ldots, F_{k}(x)(t)\right)^{T} \leq e^{-\mu_{[k]} t} \psi_{[k]}(0) \\
& +\int_{0}^{t} e^{-\mu_{[k]}(t-a)}\left(f_{1}\left(t, x_{a}\right), \ldots, f_{k}\left(t, x_{a}\right)\right)^{T} d a \\
& \leq e^{-\mu_{[k]} t} \psi_{[k]}(0)+\int_{0}^{t} e^{-\mu_{[k]}(t-a)} L\left(x_{a}^{(k)}\right) d a \\
& \leq e^{-\mu_{[k]} t} \psi_{[k]}(0)+\int_{0}^{t} e^{-\mu_{[k]}(t-a)} L\left(v_{a}^{(k)}\right) d a \leq\left(v_{1}(t), \ldots, v_{k}(t)\right)^{T}, \quad t \in[0, \tau] .
\end{aligned}
$$

Следовательно, для любой функции $x \in C_{\psi, 0, v}$ верно $F(x) \in C_{\psi, 0, v}$. Параметры $v(t)$ не зависят от $\tau$. Учитывая произвольность выбора $\tau$, завершаем доказательство леммы.

Теорема. Пусть выполнены условия, перечисленные в лемме 2. Тогда задача Коши (1), (2) имеет на промежутке $[0, \infty)$ единственное решение $x(t)$ и для всех $t \in I_{\omega} \cup[0, \infty)$ справедливы покомпонентные оценки

$$
\begin{gathered}
0 \leq x_{i}(t) \leq c_{i} e^{-r t}, \quad 1 \leq i \leq k, \\
0 \leq x_{j}(t) \leq \eta_{j}, \quad k+1 \leq j \leq m,
\end{gathered}
$$

где $c=\left(c_{1}, \ldots, c_{k}\right)^{T}$ и $r$ удовлетворяют неравенствам (14), (15), $\eta_{k+1}, \ldots, \eta_{m}$ заданы формулой (6).

ДоКАЗАТЕЛЬСтво. Используя условия теоремы и утверждения лемм 1,2 , получаем, что для любого фиксированного $\tau>0$ множество $C_{\psi, 0, v}$ инвариантно для оператора $F$. Применяя лемму 2 из [3], устанавливаем, что задача (3), (4) имеет на промежутке $[0, \tau]$ единственное решение $x=x(t)$, причем $x \in C_{\psi, 0, v}$. Учитывая произвольность выбора $\tau$ и тот факт, что компоненты функции $v(t)$ не зависят от $\tau$, получаем, что задача $(3),(4)$ имеет единственное решение $x=$ $x(t)$ на промежутке $[0, \infty)$ и для этого решения имеют место оценки $(19),(20)$. Теорема доказана.

3. Пример. Рассмотрим математическую модель, описывающую распространение инфекции среди населения некоторого региона. Переменные модели таковы: $x_{1}(t)$ - численность латентно-инфицированных (не заразных) индивидуумов, $x_{2}(t)$ - численность больных (заразных) индивидуумов, $x_{3}(t)$ - численность восприимчивых к инфекции индивидуумов. Уравнения модели имеют следующий вид:

$$
\begin{gathered}
\frac{d x_{1}(t)}{d t}=\sigma_{1} \beta x_{2}\left(t-\omega_{1}\right) x_{3}\left(t-\omega_{1}\right)-\left(\lambda_{1}+\gamma\right) x_{1}(t)+\sigma_{3} \eta x_{2}\left(t-\omega_{3}\right), \\
\frac{d x_{2}(t)}{d t}=\sigma_{2} \beta x_{2}\left(t-\omega_{2}\right) x_{3}\left(t-\omega_{2}\right)+\gamma x_{1}(t)-\left(\lambda_{2}+\eta\right) x_{2}(t), \quad t \geq 0, \\
\frac{d x_{3}(t)}{d t}=\rho(t)-\lambda_{3} x_{3}(t)-\beta x_{2}(t) x_{3}(t), \\
x_{1}(0)=x_{1}^{(0)}, x_{2}(t)=x_{2}^{(0)}(t), x_{3}(t)=x_{3}^{(0)}(t), t \in I_{\omega}=\left[-\max \left\{\omega_{1}, \omega_{2}, \omega_{3}\right\}, 0\right] .
\end{gathered}
$$


В начальных данных $(24)$ функции $x_{2}^{(0)}(t), x_{3}^{(0)}(t)$ неотрицательны и непрерывны, $x_{1}^{(0)} \geq 0$. Функция $\rho(t)$ неотрицательна, непрерывна и ограничена сверху константой $\rho^{*}>0, t \in[0, \infty)$. Все параметры, входящие в уравнения $(21)-(23)$, положительны. Функция $\rho(t)$ означает скорость пополнения группы восприимчивых индивидуумов. Параметры $\lambda_{1}, \lambda_{2}, \lambda_{3}$ задают интенсивность смертности индивидуумов и интенсивность их миграционных оттоков в другие регионы. Параметр $\beta$ отражает интенсивность контактов восприимчивых и больных индивидуумов. Параметры $\sigma_{1}>0, \sigma_{2}>0, \sigma_{1}+\sigma_{2}<1$, учитывают доли восприимчивых индивидуумов, которые после инфицирования перешли в латентную или активную стадию заболевания. Продолжительности таких переходов (считая от момента инфицирования) описываются постоянными запаздываниями $\omega_{1}$ и $\omega_{2}$. Параметр $\gamma$ означает интенсивность спонтанного развития заболевания для латентно-инфицированных индивидуумов. Параметр $\eta$ задает интенсивность перехода больных индивидуумов в неинфекционную стадию заболевания вследствие начала процесса самолечения или лечения, получаемого в медицинских учреждениях. Продолжительность пребывания в этой стадии описывается постоянным запаздыванием $\omega_{3}$. После пребывания в неинфекционной стадии заболевания доля $0<\sigma_{3}<1$ ранее поступивших индивидуумов пополняет группу латентно-инфицированных индивидуумов.

Отметим, что параметр $\eta$ может учитывать интенсивность процесса выявления больных индивидуумов с помощью различных средств диагностики заболевания. Функция $\rho(t)$ и параметр $\beta$ могут изменяться в зависимости от различных мероприятий, проводимых системой здравоохранения региона.

Соотношения (21)-(24) можно рассматривать как один из вариантов математической модели распространения туберкулеза. В отличие от известных моделей $[11,12]$ уравнения $(21)-(23)$ можно дополнить переменными $w_{1}(t), w_{2}(t)$, отражающими пребывание индивидуумов в промежуточных (скрытых) стадиях развития инфекционного процесса, а также переменной $w_{3}(t)$, описывающей численность больных индивидуумов в неинфекционной стадии заболевания:

$$
\begin{aligned}
& w_{1}(t)=\int_{t-\omega_{1}}^{t} e^{-\lambda_{3}(t-a)} \nu_{1} \beta x_{2}(a) x_{3}(a) d a, \\
& w_{2}(t)=\int_{t-\omega_{2}}^{t} e^{-\lambda_{3}(t-a)} \nu_{2} \beta x_{2}(a) x_{3}(a) d a, \\
& w_{3}(t)=\int_{t-\omega_{3}}^{t} e^{-\lambda_{3}(t-a)} \eta x_{2}(a) d a, \quad t \geq 0,
\end{aligned}
$$

где $\nu_{1}>0, \nu_{2}>0, \nu_{1}+\nu_{2}=1-$ некоторые константы. Для вывода приведенных соотношений использованы работы $[13,14]$.

Интегральные соотношения для переменных $w_{1}(t), w_{2}(t), w_{3}(t)$ можно свести к задаче Коши следующего вида:

$$
\begin{aligned}
& \frac{d w_{1}(t)}{d t}=\nu_{1} \beta x_{2}(t) x_{3}(t)-\lambda_{3} w_{1}(t)-e^{-\lambda_{3} \omega_{1}} \nu_{1} \beta x_{2}\left(t-\omega_{1}\right) x_{3}\left(t-\omega_{1}\right), \\
& \frac{d w_{2}(t)}{d t}=\nu_{2} \beta x_{2}(t) x_{3}(t)-\lambda_{3} w_{2}(t)-e^{-\lambda_{3} \omega_{2}} \nu_{2} \beta x_{2}\left(t-\omega_{2}\right) x_{3}\left(t-\omega_{2}\right),
\end{aligned}
$$




$$
\begin{gathered}
\frac{d w_{3}(t)}{d t}=\eta x_{2}(t)-\lambda_{3} w_{3}(t)-e^{-\lambda_{3} \omega_{3}} \eta x_{2}\left(t-\omega_{3}\right), \quad t \geq 0 \\
w_{1}(0)=\int_{-\omega_{1}}^{0} e^{\lambda_{3} a} \nu_{1} \beta x_{2}^{(0)}(a) x_{3}^{(0)}(a) d a, \quad w_{2}(0)=\int_{-\omega_{2}}^{0} e^{\lambda_{3} a} \nu_{2} \beta x_{2}^{(0)}(a) x_{3}^{(0)}(a) d a \\
w_{3}(0)=\int_{-\omega_{3}}^{0} e^{\lambda_{3} a} \eta x_{2}^{(0)}(a) d a .
\end{gathered}
$$

Учитывая структуру уравнений для $w_{i}(t), i=1,2,3$, можно записать, что в уравнениях $(21),(22)$ параметры $\sigma_{1}, \sigma_{2}, \sigma_{3}$ таковы:

$$
\sigma_{1}=e^{-\lambda_{3} \omega_{1}} \nu_{1}, \quad \sigma_{2}=e^{-\lambda_{3} \omega_{2}} \nu_{2}, \quad \sigma_{3}=e^{-\lambda_{3} \omega_{3}} .
$$

Система уравнений (21)-(23) с начальными данными (24) соответствует задаче Коши (1), (2). Правые части системы (21)-(23) удовлетворяют условиям, указанным в предположении (Н0). Примем дополнительно, что

$$
x_{3}^{(0)}(t) \leq x_{3}^{(*)}=\rho^{*} / \lambda_{3}, \quad t \in I_{\omega} .
$$

Исходя из структуры правых частей уравнений изучаемой системы, получаем, что для неотрицательных непрерывных функций $x_{1}(t), x_{2}(t), x_{3}(t)$ верны следующие оценки: $0 \leq x_{3}(t) \leq x_{3}^{*}, t \in I_{\omega} \cup[0, \infty)$,

$$
\begin{gathered}
\frac{d x_{1}(t)}{d t} \leq \sigma_{1} \beta x_{3}^{*} x_{2}\left(t-\omega_{1}\right)-\left(\lambda_{1}+\gamma\right) x_{1}(t)+\sigma_{3} \eta x_{2}\left(t-\omega_{3}\right), \\
\frac{d x_{2}(t)}{d t} \leq \sigma_{2} \beta x_{3}^{*} x_{2}\left(t-\omega_{2}\right)+\gamma x_{1}(t)-\left(\lambda_{2}+\eta\right) x_{2}(t), \\
\frac{d x_{3}(t)}{d t} \leq \rho^{*}-\lambda_{3} x_{3}(t), \quad t \geq 0 .
\end{gathered}
$$

Следовательно, можно записать вектор $p$ и матрицы $L_{k, i}, L_{k}, \mu_{[k]}-L_{[k]}$, возникающие в предположении (H1) при $m=3, k=2, k+1=3$, а именно:

$$
\begin{gathered}
p=p_{3}=\rho^{*}, \quad f_{3}\left(t, x_{t}\right) \leq p_{3}=\rho^{*}, \\
\mu_{[2]}=\left(\begin{array}{cc}
\lambda_{1}+\gamma & 0 \\
0 & \lambda_{2}+\eta
\end{array}\right), \quad L_{2,0}=\left(\begin{array}{cc}
0 & 0 \\
\gamma & 0
\end{array}\right), \quad L_{2,1}=\left(\begin{array}{cc}
0 & \sigma_{1} \beta x_{3}^{*} \\
0 & 0
\end{array}\right), \\
L_{2,2}=\left(\begin{array}{cc}
0 & 0 \\
0 & \sigma_{2} \beta x_{3}^{*}
\end{array}\right), \quad L_{2,3}=\left(\begin{array}{cc}
0 & \sigma_{3} \eta \\
0 & 0
\end{array}\right), \quad L_{2,4}(\theta) \equiv 0, \theta \in I_{\omega}, \\
L_{[2]}=\left(\begin{array}{cc}
0 & \sigma_{1} \beta x_{3}^{*}+\sigma_{3} \eta \\
\gamma & \sigma_{2} \beta x_{3}^{*}
\end{array}\right), \quad \mu_{[2]}-L_{[2]}=\left(\begin{array}{cc}
\lambda_{1}+\gamma & -\sigma_{1} \beta x_{3}^{*}-\sigma_{3} \eta \\
-\gamma & \lambda_{2}+\eta-\sigma_{2} \beta x_{3}^{*}
\end{array}\right) .
\end{gathered}
$$

Обращаясь к условиям теоремы, потребуем, чтобы $\mu_{[2]}-L_{[2]}$ являлась невырожденной М-матрицей. Применим критерий, состоящий в том, что все главные миноры $\mu_{[2]}-L_{[2]}$ должны быть положительны [9, гл. 6, п. 2]. Первый такой минор равен $\lambda_{1}+\gamma$ и, очевидно, положителен. Второй главный минор совпадает с определителем рассматриваемой матрицы и имеет вид

$$
\operatorname{det}\left(\mu_{[2]}-L_{[2]}\right)=\left(\lambda_{1}+\gamma\right)\left(\lambda_{2}+\eta\right)\left(1-R_{0}\right),
$$


где

$$
R_{0}=\frac{\sigma_{3} \gamma \eta}{\left(\lambda_{1}+\gamma\right)\left(\lambda_{2}+\eta\right)}+\frac{\beta\left(\lambda_{1} \sigma_{2}+\gamma\left(\sigma_{1}+\sigma_{2}\right)\right)}{\left(\lambda_{1}+\gamma\right)\left(\lambda_{2}+\eta\right)} x_{3}^{*}
$$

Полагая

$$
R_{0}<1
$$

получаем, что $\mu_{[2]}-L_{[2]}$ является невырожденной М-матрицей.

Следовательно, если выполнены неравенства (25) и (26), то для компонент $x_{1}(t), x_{2}(t)$ решения модели $(21)-(24)$ справедливы оценки

$$
0 \leq x_{1}(t) \leq c_{1} e^{-r t}, \quad 0 \leq x_{2}(t) \leq c_{2} e^{-r t}, \quad t \in I_{\omega} \cup[0, \infty) .
$$

Положим $c_{[2]}=\left(c_{1}, c_{2}\right)^{T}, \psi_{[2]}(t)=\left(x_{1}^{(0)}, x_{2}^{(0)}(t)\right)^{T}, t \in I_{\omega}$,

$$
H(r)=\left(h_{i j}(r)\right)=\mu_{[2]}-r I_{[2]}-L_{2,0}-e^{r \omega_{1}} L_{2,1}-e^{r \omega_{2}} L_{2,2}-e^{r \omega_{3}} L_{2,3}, \quad r \in R .
$$

Для нахождения констант $c_{1}, c_{2}, r$, входящих в оценки $(27)$, используем формулы (14), (15), которые приводят к следующим соотношениям:

$$
\begin{gathered}
c_{[2]}>0, \quad H(r) c_{[2]} \geq 0, \\
c_{[2]} \geq \max _{t \in I_{\omega}}\left(e^{r t} \psi_{[2]}(t)\right), \quad 0<r<\min \left(\lambda_{1}+\gamma, \lambda_{2}+\eta\right) .
\end{gathered}
$$

Следуя [2], константу $r$ будем искать как положительный корень уравнения

$$
\operatorname{det} H(r)=\left(\lambda_{1}+\gamma-r\right)\left(\lambda_{2}+\eta-r-e^{r \omega_{2}} \sigma_{2} \beta x_{3}^{*}\right)-\gamma\left(e^{r \omega_{1}} \sigma_{1} \beta x_{3}^{*}+e^{r \omega_{3}} \sigma_{3} \eta\right)=0 \text {. }
$$

Заметим, что $H(0)=\mu_{[2]}-L_{[2]}$ и $\operatorname{det} H(0)>0$ в силу неравенства $(26)$. Используя (30), перейдем к уравнению относительно искомой константы $r$ :

$$
\lambda_{2}+\eta-r-e^{r \omega_{2}} \sigma_{2} \beta x_{3}^{*}=\gamma \frac{e^{r \omega_{1}} \sigma_{1} \beta x_{3}^{*}+e^{r \omega_{3}} \sigma_{3} \eta}{\lambda_{1}+\gamma-r}, 0 \leq r<\min \left(\lambda_{1}+\gamma, \lambda_{2}+\eta\right) \text {. }
$$

Видно, что левая часть уравнения (31) представляет собой убывающую функцию $\varphi_{1}(r)$, тогда как правая часть - возрастающую функцию $\varphi_{2}(r)$. Поскольку $\operatorname{det} H(0)>0$, то $\varphi_{1}(0)>\varphi_{2}(0)$. Следовательно, существует единственный корень $0<r_{*}<\min \left(\lambda_{1}+\gamma, \lambda_{2}+\eta\right)$ уравнения (31) и соответственно уравнения (30).

Для нахождения вектора $c_{[2]}$ поступим следующим образом. Опираясь на (28), рассмотрим уравнение $H\left(r_{*}\right) u=0$, где $u=\left(u_{1}, u_{2}\right)^{T} \in R^{2}$ :

$$
h_{11}\left(r_{*}\right) u_{1}+h_{12}\left(r_{*}\right) u_{2}=0, \quad h_{21}\left(r_{*}\right) u_{1}+h_{22}\left(r_{*}\right) u_{2}=0 .
$$

Так как $\operatorname{det} H\left(r_{*}\right)=0$ и $h_{11}\left(r_{*}\right)=\lambda_{1}+\gamma-r_{*} \neq 0$, искомый $и$ имеет вид

$$
u=\left(u_{1}, u_{2}\right)^{T}=\alpha u^{*}=\alpha\left(u_{1}^{*}, 1\right)^{T},
$$

где $\alpha \in R$ - произвольная константа, а компонента $u_{1}^{*}$ такова:

$$
u_{1}^{*}=\left(e^{r_{*} \omega_{1}} \sigma_{1} \beta x_{3}^{*}+e^{r_{*} \omega_{3}} \sigma_{3} \eta\right) /\left(\lambda_{1}+\gamma-r_{*}\right) .
$$

Полагая $\alpha>0$, устанавливаем, что $r_{*}$ и вектор $c_{[2]}=\alpha u^{*}$ удовлетворяют (28). Вектор $c_{[2]}$ можно выбрать как $c_{[2]}=\alpha_{*} u^{*}$, где константа $\alpha_{*}>0$ подбирается из условия выполнения неравенств (29) и, очевидно, зависит от компонент начальной функции $\psi_{[2]}(t)$. 
Заметим, что для дополнительных переменных $w_{1}(t), w_{2}(t), w_{3}(t)$ верны оценки, аналогичные (27), а именно:

$$
0 \leq w_{i}(t) \leq d_{i} e^{-r t}, \quad i=1,2,3, t \in[0, \infty),
$$

где константы $d_{1}>0, d_{2}>0, d_{3}>0$ выражаются через $c_{2}, x_{3}^{(*)}$ и параметры, входящие в интегральные выражения для указанных переменных.

В рамках рассматриваемой модели неравенства $(25),(26)$ интерпретируются как условия экспоненциального затухания эпидемического процесса среди населения изучаемого региона. Следуя принятой терминологии $[15,16]$, константу $R_{0}$, входящую в неравенство (26), назовем базовым репродуктивным числом. Константа $R_{0}$ отражает среднее количество больных (заразных) индивидуумов, воспроизводимых популяцией в расчете на одного латентно-инфицированного индивидуума, одного больного индивидуума и $x_{3}^{*}$ восприимчивых индивидуумов.

Используя выражения для $R_{0}$ и параметров $\sigma_{1}, \sigma_{2}, \sigma_{3}$, получаем оценку

$$
R_{0}<\widehat{R}_{0}=\frac{\gamma e^{-\lambda_{3} \omega_{3}}}{\lambda_{1}+\gamma}+\frac{\beta\left(\gamma \nu_{1} e^{-\lambda_{3} \omega_{1}}+\left(\lambda_{1}+\gamma\right) \nu_{2} e^{-\lambda_{3} \omega_{2}}\right) x_{3}^{*}}{\left(\lambda_{1}+\gamma\right)\left(\lambda_{2}+\eta\right)} .
$$

Из (33) видно, что в каждом наборе параметров модели найдутся достаточно большие $\omega_{3}, \eta$ и достаточно малые $\gamma, \beta, x_{3}^{*}$, для которых в том или ином сочетании будет выполнено неравенство $\widehat{R}_{0}<1$. Значения указанных параметров и оценки (27), (32) могут быть использованы при планировании работы системы здравоохранения региона по выявлению и лечению больных индивидуумов, а также по искоренению инфекции в течение некоторого периода времени.

\section{ЛИТЕРАТУРА}

1. Перцев Н. В. Применение М-матриц для построения экспоненциальных оценок решений задачи Коши для некоторых систем линейных разностных и дифференциальных уравнений // Мат. тр. 2013. Т. 16, № 2. С. 111-141.

2. Перцев Н. В. Двусторонние оценки на решения задачи Коши для систем линейных дифференциальных уравнений Важевского с запаздыванием // Сиб. мат. журн. 2013. Т. 54, № 6. C. $1368-1379$.

3. Перцев Н. В. Глобальная разрешимость и оценки решений задачи Коши для функционально-дифференциальных уравнений с запаздыванием, используемых в моделях живых систем // Сиб. мат. журн. 2018. Т. 59, № 1. С. 143-157.

4. Красносельский М. А., Вайникко Г. М., Забрейко П. П., Рутицкий Я. Б., Стеценко В. Я. Приближенное решение операторных уравнений. М.: Наука, 1969.

5. Цалюк 3. Б. Интегральные уравнения Вольтерра // Математический анализ. М.: ВИНИТИ, 1977. Т. 15. С. 131-198. (Итоги науки и техники).

6. Азбелев Н. В., Максимов В. П., Рахматуллина Л. Ф. Элементы современной теории функционально-дифференциальных уравнений. Методы и приложения. М.: Ин-т компьютерных исследований, 2002.

7. Хейл Дж. Теория функционально-дифференциальных уравнений. М.: Мир, 1984.

8. Колмановский В. Б., Носов В. Р. Устойчивость и периодические режимы регулируемых систем с последействием. М.: Наука, 1981.

9. Berman A., Plemmons R. J. Nonnegative matrices in the mathematical sciences. New York: Acad. Press, 1979.

10. Воеводин В. В., Кузнецов Ю. А. Матрицы и вычисления. М.: Наука, 1984.

11. Avilov K. K., Romanyukha A. A. Mathematical modelling of tuberculosis propagation and patient detection // Automation Remote Control. 2007. V. 68, N 9. P. 1604-1617.

12. Перцев Н. В. Непрерывно-дискретная модель распространения и контроля туберкулеза // Сиб. журн. индустр. математики. 2014. Т. 17, № 3. С. 86-97.

13. Yuan Y., Belair J. Threshold dynamics in an SEIRS model with latency and temporary immunity // J. Math. Biol. 2014. V. 69. P. 86-97. 
14. Перцев Н. В. Об условиях корректности интегральных моделей некоторых живых систем // Дифференц. уравнения. 2017. Т. 53, № 9. С. 1162-1179.

15. Hethcote H. The mathematics of infectious diseases // SIAM Rev. 2000. V. 42. P. 599-653.

16. Андерсон P., Мэй Р. Инфекционные болезни человека. Динамика и контроль. М.: Мир, 2004.

Поступила в редакиию 27 нолбря 2019 г.

После доработки 4 мая 2020 г.

Принята к публикации 17 июня 2020 г.

Перцев Николай Викторович

Институт математики им. С. Л. Соболева СО РАН, Омский филиал, ул. Певцова, 13, Омск 644043

homlab@ya.ru 